\title{
Correction to: Spindle-related brain activation in patients with insomnia disorder: An EEG-fMRI study
}

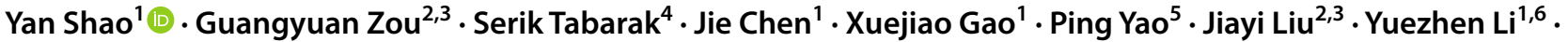 \\ Nana Xiong ${ }^{1}$. Wen Pan ${ }^{7} \cdot$ Mengying $\mathrm{Ma}^{1}$. Shuqin $\mathrm{Zhou}^{3}$. Jing $\mathrm{Xu}^{3}$. Yundong Ma ${ }^{1}$. Jiahui Deng ${ }^{1}$ - Qiqing Sun ${ }^{1}$. \\ Yanping $\mathrm{Bao}^{8} \cdot$ Wei Sun $^{1} \cdot \mathrm{Jie} \mathrm{Shi}^{8} \cdot$ Qihong Zou ${ }^{1,3} \cdot \mathrm{Jia}^{-H o n g} \mathrm{Gao}^{2,3,9} \cdot$ Hongqiang Sun ${ }^{1}$
}

Published online: 13 December 2021

๑) Springer Science+Business Media, LLC, part of Springer Nature 2021

\section{Correction to: Brain Imaging and Behaviors https://doi.org/10.1007/s11682-021-00544-2}

The original published version of this article contained mistakes. Some errors about authorship and affiliations listed below were correct on proofs, but there is something wrong with the online version which needs to be modified.

1. Yan Shao and Guangyuan Zou and contributed equally to this work.

2. The affiliation of 9,10 , and 11 should be changed as 1 , and 3 .

3. The affiliation of 12,13 , and 14 should be changed as 2,3 , and 9 .
4. The affiliation of 15 should be changed as 1 .

The original article has been corrected.

Publisher's note Springer Nature remains neutral with regard to jurisdictional claims in published maps and institutional affiliations.
Yan Shao and Guangyuan Zou contributed equally to this work.

The original article can be found online at https://doi.org/10.1007/ s11682-021-00544-2.

Qihong Zou

zouqihong@pku.edu.cn

$\triangle$ Jia-Hong Gao

jgao@pku.edu.cn

$\triangle$ Hongqiang Sun

sunhq@bjmu.edu.cn

1 Peking University Sixth Hospital, Peking University Institute of Mental Health, NHC Key Laboratory of Mental Health (Peking University), National Clinical Research Center for Mental Disorders (Peking University Sixth Hospital), Beijing, China

2 Beijing City Key Lab for Medical Physics and Engineering, Institute of Heavy Ion Physics, School of Physics, Peking University, Beijing, China

3 Center for MRI Research, Academy for Advanced Interdisciplinary Studies, Peking University, Beijing, China
4 Peking-Tsinghua Center for Life Sciences, PKU-IDG/McGovern Institute for Brain Research, Peking University, Beijing, China

5 Department of Physiology, College of Basic Medicine, Inner Mongolia Medical University, Hohhot, China

6 Department of Neuropsychiatry, Behavioral Neurology and Sleep Center, Beijing Tian Tan Hospital, Capital Medical University, Beijing, China

7 Sleep Medicine Center, Suzhou Guangji Hospital, The Afliated (Affiliated) Guangji Hospital of Soochow University, Suzhou, China

8 National Institute On Drug Dependence and Beijing Key Laboratory of Drug Dependence, Peking University, Beijing, China

9 McGovern Institute for Brain Research, Peking University, Beijing, China 